\title{
The Natural History of a Patient With COVID-19 Pneumonia and Silent Hypoxemia
}

Nicholas Hornstein, MD; Gilad M. Jaffe, MD; Kelley Chuang, MD; Jaime Betancourt, MD; and Guy W. Soo Hoo, MD

\begin{abstract}
A patient who declined all interventions, including oxygen, and recovered highlights the importance of treating the individual instead of clinical markers and provides a time course for recovery from pneumonia and severe hypoxemia.
\end{abstract}

\author{
Author affiliations \\ can be found at the \\ end of the article. \\ Correspondence: \\ Guy Soo Hoo \\ (guy.soohoo@va.gov) \\ Fed Pract. 2021;38(4). \\ Published online \\ April 12, 2021 \\ doi:10.12788/fp.0100
}

F $\mathrm{n}$ less than a year, COVID-19 has infected nearly 100 million people worldwide and caused more than 2 million deaths and counting. Although the infection fatality rate is estimated to be $1 \%$ and the case fatality rate between $2 \%$ and 3\%, COVID-19 has had a disproportionate effect on the older population and those with comorbidities. Some of these findings are mirrored in the US Department of Veterans Affairs (VA) population, which has seen a higher case fatality rate..$^{1-4}$

As a respiratory tract infection, the most dreaded presentation is severe pneumonia with acute hypoxemia, which may rapidly deteriorate to acute respiratory distress syndrome (ARDS) and respiratory failure..$^{5-7}$ This possibility has led to early intubation strategies aimed at preempting this rapid deterioration and minimizing viral exposure to health care workers. Intubation rates have varied widely with extremes of 6 to $88 \% .{ }^{8,9}$

However, this early intubation strategy has waned as some of the rationale behind its endorsement has been called into question. Early intubation bypasses alternatives to intubation; high-flow nasal cannula oxygen, noninvasive ventilation, and awake proning are all effective maneuvers in the appropriate patient. ${ }^{10,11}$ The use of firstline high-flow nasal cannula oxygen and noninvasive ventilation has been widely reported. Reports of first-line use of highflow nasal cannula oxygen has not demonstrated inferior outcomes, nor has the timing of intubation, suggesting a significant portion of patients could benefit from a trial of therapy and eventually avoid intubation. ${ }^{11-14}$ Other therapies, such as systemic corticosteroids, confer a mortality benefit in those patients with COVID-19 who require oxygen or mechanical ventilation, but their impact on the progression of respiratory failure and need for intubation are undetermined.

There also are reports of patients who report no signs of respiratory distress or dyspnea with their COVID-19 pneumonia despite profound hypoxemia or high oxygen requirements. Various terms, including silent hypoxemia or happy hypoxia, are descriptive of the demeanor of these patients, and treatment has invariably included oxygen. ${ }^{15,16}$ Nevertheless, low oxygen measurements have generally prompted higher levels of supplemental oxygen or more invasive therapies.

Treatment rendered may obscure the trajectory of response, which is important to understand to better position options for invasive therapies and other therapeutics. We recently encountered a patient with a course of illness that represented the natural history of COVID-19 pneumonia with low oxygen levels (referred to as hypoxemia for consistency) that highlighted several issues of management.

\section{Case Presentation}

A 62-year-old undomiciled woman with morbid obesity, prediabetes mellitus, longstanding schizophrenia, and bipolar disorder presented to our facility for evaluation of dry cough and need for tuberculosis clearance for admittance to a shelter. She appeared comfortable and was afebrile with blood pressure $111 / 74 \mathrm{~mm} \mathrm{Hg}$, heart rate 82 beats per minute. Her respiratory rate was 18 breaths per minute, but the pulse oximetry showed oxygen saturation of 70 to 
$75 \%$ on room air at rest. A chest X-ray showed bibasilar infiltrates (Figure 1), and a rapid COVID-19 nasopharyngeal polymerase chain reaction (PCR) test returned positive, confirmed by a second PCR test. Baseline inflammatory markers were elevated (Figure 2). In addition, the serum interleukin- 6 also was elevated to $66.1 \mathrm{pg} / \mathrm{mL}$ (normal < 5.0), erythrocyte sedimentation rate elevated to $69 \mathrm{~mm} / \mathrm{h}$, but serum procalcitonin was essentially normal $(0.22 \mathrm{ng} / \mathrm{mL}$; normal $<20 \mathrm{ng} / \mathrm{mL}$ ) as was the serum lactate $(1.4 \mathrm{mmol} / \mathrm{L})$.

The patient was admitted to the intensive care unit (ICU) for close monitoring in anticipation of the possibility of decompensation based on her age, hypoxia, and elevated inflammatory markers. ${ }^{17} \mathrm{Be}$ sides a subsequent low-grade fever $\left(100.4^{\circ} \mathrm{F}\right)$ and lymphopenia (manual count $550 / \mathrm{uL}$ ), she remained clinically unchanged. Throughout her hospitalization, she maintained a persistent psychotic delusion that she did not have COVID-19, refusing all medical interventions, including a peripheral IV line and supplemental oxygen for the entire duration. Extensive efforts to identify family or a surrogate decision maker were unsuccessful. After consultation with Psychiatry, BioEthics, and hospital leadership, the patient was deemed to lack decision-making capacity regarding treatment or disposition and was placed on a psychiatric hold. However, since any interventions against her will would require sedation, IV access, and potentially increase the risk of nosocomial COVID-19 transmission, she was allowed to remain untreated and was closely monitored for symptoms of worsening respiratory failure.

Over the next 2 weeks, her hypoxemia, inflammatory markers, and the infiltrates on imaging resolved (Figure 2 ). The lowest daily awake room air pulse oximetry readings are reported, initially with consistent readings
FIGURE 1 Admission and Discharge Chest X-rays

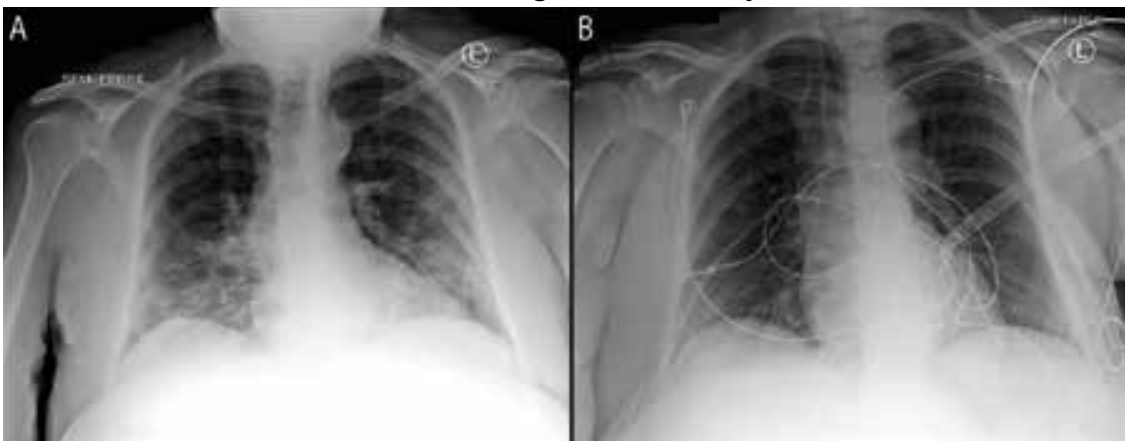

A, On admission, findings were consistent with COVID-19 pneumonia, including bibasilar infiltrates. B, Prior to discharge, improvement of infiltrates can be seen.

\section{FIGURE 2 Trends in Peripheral Capillary Oxygen Saturation and} Inflammatory Markers

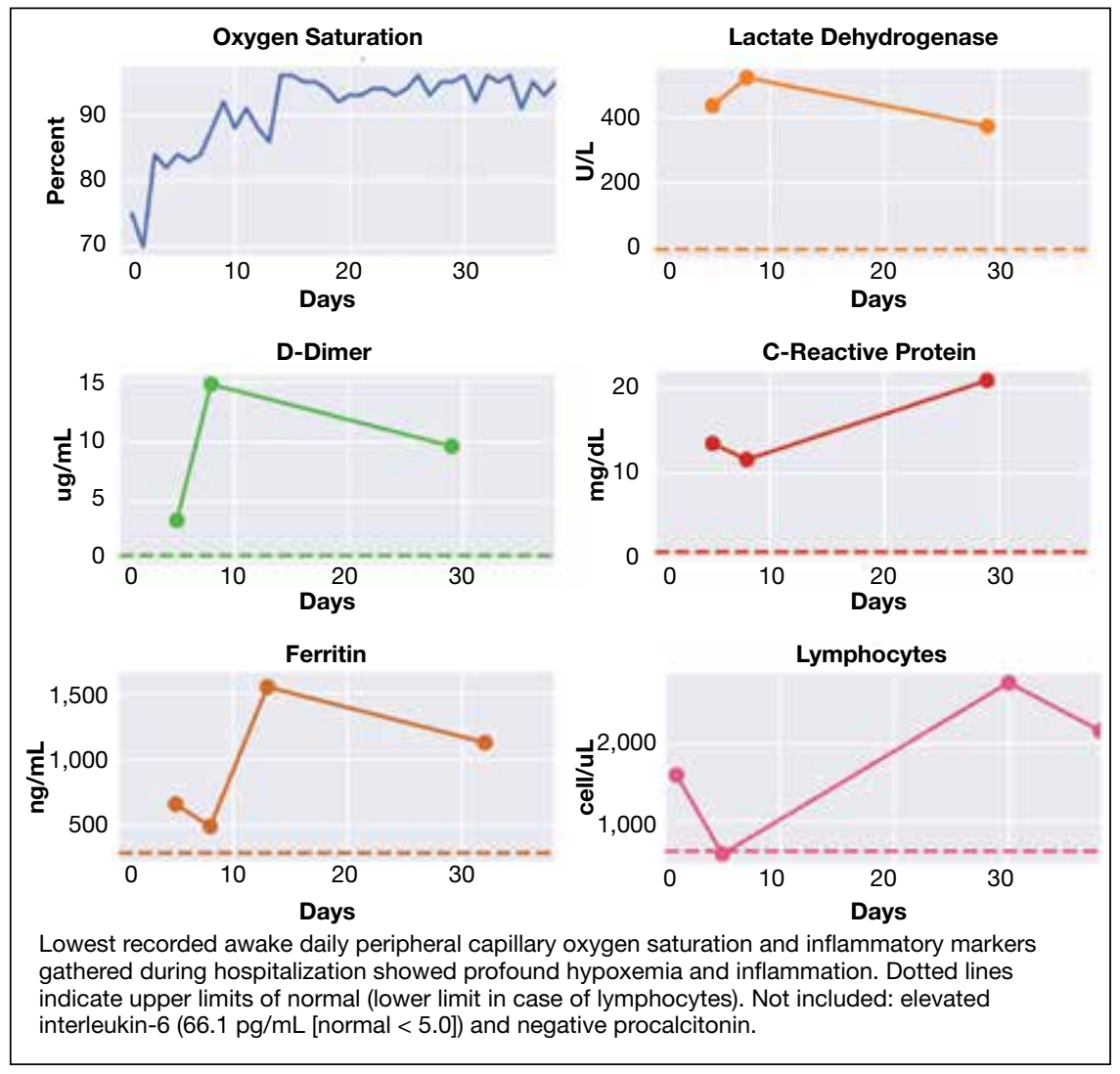

in the low $80 \%$ range, but on day 12 , readings were $>90 \%$ and remained $>90 \%$ for the remainder of her hospitalization. Therefore, shortly after hospital day 12 , she was clinically stable for discharge from acute care to a subacute facility, but this required documentation of the clearance of her viral infection. She refused to undergo a subsequent nasopharyngeal swab but allowed an 
TABLE 1 Pulse Oximetry $\mathrm{PaO}_{2}$ Conversion

\begin{tabular}{lc}
$\begin{array}{l}\text { Pulse } \\
\text { Oximetry, \% }\end{array}$ & $\begin{array}{c}\text { Partial Pressure of } \mathrm{O}_{2} \\
\text { Under Ideal Circumstances, } \mathbf{m m ~ H g}\end{array}$ \\
\hline 90 & 60 \\
\hline 85 & 50 \\
\hline 80 & 44 \\
\hline 75 & 40 \\
\hline
\end{tabular}

oropharyngeal COVID-19 PCR swab, which was negative. She remained stable and unchanged for the remainder of her hospitalization, awaiting identification of a receiving facility and was able to be discharged to transitional housing on day 38 .

\section{DISCUSSION}

The initial reports of COVID-19 pneumonia focused on ARDS and respiratory failure requiring mechanical ventilation with less emphasis on those with lower severity of illness. This was heightened by health care systems that were overwhelmed with large number of patients while faced with limited supplies and equipment. Given the risk to patients and providers of crash intubations, some recommended early intubation strategies. ${ }^{3}$ However, the natural history of COVID-19 pneumonia and the threshold for intubation of these patients remain poorly defined despite the creation of prognostic tools. ${ }^{17}$ This patient's persistent hypoxemia and elevated inflammatory markers certainly met markers of disease associated with a high risk of progression.

The greatest concern would have been her level of hypoxemia. Acceptable thresholds of hypoxemia vary, but general consensus would classify pulse oximetry $<90 \%$ as hypoxemia and a threshold for administering supplemental oxygen. It is important to recognize how pulse oximetry readings translate to partial pressure of oxygen $\left(\mathrm{PaO}_{2}\right)$ measurements (Table 1 ). Pulse oximetry readings of $90 \%$ corresponds to a $\mathrm{PaO}_{2}$ readings of $60 \mathrm{~mm} \mathrm{Hg}$ in ideal conditions without the influence of acidosis, $\mathrm{PaCO}_{2}$, or temperature. While lower readings are of concern, these do not represent absolute indications for assisted ventilatory support as lower levels are well tolerated in a variety of conditions. A common example are patients with chronic obstructive pulmonary disease. Long-term mortality benefits of continuous supplemental oxygen are well established in specific populations, but the threshold for correction in the acute setting remains a case-bycase decision. This decision is complex and is based on more than an absolute number or the amount of oxygen required to achieve a threshold level of oxygenation.

The $\mathrm{PaO}_{2} / \mathrm{FIO}_{2}$ (fraction of inspired oxygen) is a common measure used to address severity of disease and oxygen requirements. It also has been used to define the severity of ARDS, but the ratio is based on intubated and mechanically ventilated patients and may not translate well to those not on assisted ventilation. Treatment with supplemental oxygen also involves entrained air with associated imprecision in oxygen delivery. ${ }^{18}$ For this discussion, the patient's admission $\mathrm{PaO}_{2} / \mathrm{FIO}_{2}$ on room air would have been between 190 and 260. Coupled with the bilateral infiltrates on imaging, there was justified concern for progression to severe ARDS. Her presentation would have met most of the epidemiologic criteria used in initial case finding for severe COVID-19 cases, including a blood oxygen saturation $\leq 93 \%, \mathrm{PaO}_{2} / \mathrm{FIO}_{2}$ $<300$ with infiltrates involving close to if not exceeding $50 \%$ of the lung.

With COVID-19 pneumonia, the pathologic injury to the alveoli resembles that of any viral pneumonia with recruitment of predominantly lymphocytic inflammatory cells that fill the alveoli, derangements in ventilation/perfusion mismatch as the core mechanism of hypoxemia with interstitial edema and shuntlike physiology developing at the extremes of involvement. In later stages, the histologic appearance is similar to ARDS, including hyaline membrane formation and thickened alveolar septa with perivascular lymphocytic-plasmocytic infiltration. In addition, there also are findings of organizing pneumonia with fibroblastic proliferation, thrombosis, and diffuse alveolar damage, a constellation of findings similar to that seen in the latter stages of ARDS. ${ }^{2}$

Although these histologic findings resemble ARDS, many patients with respiratory failure due to COVID-19 have a different physiologic profile compared with those with 
typical ARDS, with the most striking finding of lungs with low elastance or high compliance. From the critical care standpoint, this meant that the lungs were relatively easy to ventilate with lower peak airway and plateau pressures and low driving pressures. This condition suggested that there was relatively less lung that could be recruited with positive end expiratory pressure; therefore, a somewhat different entity from that associated with ARDS. ${ }^{19}$ These findings were often noted early in the course of respiratory failure, and although there is debate about whether this represents a different phenotype or timepoint in the spectrum of disease, it clearly represents a subset that is distinct from that which had been previously encountered.

On the other hand, the clinical features seen in those patients with COVID-19 pneumonia who progressed to advanced respiratory failure were essentially indistinguishable from those patients with traditional ARDS. Other explanations for this respiratory failure have included a disrupted vasoregulatory response to hypoxemia with failed hypoxic vasoconstriction, intravascular microthrombi, and impaired diffusion, all contributing to impaired gas exchange and hypoxemia. ${ }^{19-21}$ This can lead to shuntlike conditions that neither respond well to supplemental oxygen nor manifest the type of physiologic response seen with other causes of hypoxemia.

The severity of hypoxemia manifested by this patient may have elicited additional findings of respiratory distress, such as dyspnea and tachypnea. However, in patients with severe COVID-19 pneumonia, dyspnea was not a universal finding, reported in the 20 to $60 \%$ range of cohorts, higher in those with ARDS and mechanical ventilation, although some report near universal dyspnea in their series. ${ }^{1,4,8,22,23}$ Tachypnea is another symptom of interest. Using a threshold of $>24$ breaths/min, tachypnea was noted in 16 to $29 \%$ of patients with a much greater proportion (63\%) in nonsurvivors. ${ }^{6,24}$ Several explanations have been proposed for the discordance between the presence and severity of hypoxemia and lack of symptoms of dyspnea and tachypnea. It is important to recognize that misclassification of the severity of hypoxemia can occur due to technical issues and potential errors involving pulse oxime-
TABLE 2 Considerations in Patients With COVID-19 Pneumonia and Silent Hypoxemia

\begin{tabular}{|c|c|}
\hline Conditions & Descriptions \\
\hline $\begin{array}{l}\text { Oxyhemoglobin } \\
\text { dissociation } \\
\text { curve }\end{array}$ & $\begin{array}{l}\text { - Shifts to the right during fever, acidosis } \\
\text { - Shifts to the left with alkalosis and hypocapnia }\end{array}$ \\
\hline $\begin{array}{l}\text { Mechanisms of } \\
\text { hypoxemia }\end{array}$ & $\begin{array}{l}\text { - Ventilation-perfusion mismatch (pneumonia, microthrombi) } \\
\text { - Shunt (disordered vasoregulation) } \\
\text { - Diffusion impairment (alveolar injury and damage) }\end{array}$ \\
\hline $\begin{array}{l}\text { Histology of } \\
\text { COVID-19 } \\
\text { pneumonia }\end{array}$ & $\begin{array}{l}\text { - Diffuse alveolar damage } \\
\text { - Interstitial edema } \\
\text { - Organizing pneumonia } \\
\text { - Vasculature with microthrombi }\end{array}$ \\
\hline $\begin{array}{l}\text { Blunted ventilatory } \\
\text { response to } \mathrm{O}_{2}\end{array}$ & $\begin{array}{l}\text { - Decreased due to age } \\
\text { - Decreased due to comorbidities (diabetes mellitus, } \\
\text { chronic obstructive pulmonary disease) } \\
\text { - Suppressed by hypocapnia }\end{array}$ \\
\hline $\begin{array}{l}\text { COVID-19 } \\
\text { pneumonia } \\
\text { physiology }\end{array}$ & $\begin{array}{l}\text { - Early: normal peak airway, plateau, driving pressures } \\
\text { - Early: normal lung compliance } \\
\text { - Late: increased peak airway, plateau, driving pressures }\end{array}$ \\
\hline $\begin{array}{l}\text { Preintubation } \\
\text { treatment of } \\
\text { hypoxemia }\end{array}$ & $\begin{array}{l}\text { - Supplemental oxygen } \\
\text { - High-flow nasal cannula oxygen } \\
\text { - Noninvasive ventilation } \\
\text { - Awake prone positioning }\end{array}$ \\
\hline
\end{tabular}

try measurement and shifts in the oxyhemoglobin dissociation curve. However, this is more pertinent for those with mild disease as the severity of hypoxemia in severe pneumonia is beyond what can be attributed to technical issues.

More important, the ventilatory response curve to hypoxemia may not be normal for some patients, blunted by as much as $50 \%$ in older patients, especially in those with diabetes mellitus. ${ }^{7,25,26}$ In addition, the ventilatory response varies widely even among normal individuals. This would translate to lower levels of minute ventilation (less tachypnea or respiratory effort) with hypoxemia. Hypocapnic hypoxemia also blunts the ventilatory response to hypoxemia. Subjects do not increase their minute ventilation if the $\mathrm{PaCO}_{2}$ remains low despite oxygen desaturation to $<70 \%$, especially if $\mathrm{PaCO}_{2}$ $<30 \mathrm{~mm} \mathrm{Hg}$ or alternatively, increases in minute ventilation are not seen until the $\mathrm{PaCO}_{2}$ exceeds $39 \mathrm{~mm} \mathrm{Hg.}{ }^{27}$ Both scenarios occur in those with COVID-19 pneumonia and provide another explanation for the 
absence of respiratory symptoms or signs of respiratory distress in some patients.

The observation of more compliant lungs may help in the understanding of the variable presentation of these patients. Compliant lungs do not require the increased pressure needed to achieve a specific tidal volume that, in turn, may increase the work of breathing. This may add to the explanation of seemingly paradoxical silent hypoxemia in those patients where the combination of a blunted ventilatory response, hypocapnia, shunt physiology, and normal respiratory system compliance is represented by the absence of increased breathing effort despite severe hypoxemia.

If not for the patient's refusal of medical services, this patient quite possibly would have been intubated due to hypoxemia and health care providers' concern for her risk of deterioration. Reported intubation and mechanical ventilation rates have varied widely from extremes of from $<5$ to $88 \%$ in severely ill patients. ${ }^{9,22}$ About $75 \%$ will need oxygen, but many can be treated and recover without the need for intubation and mechanical ventilation.

As previously mentioned, options for treatment include standard and high-flow oxygen delivery, noninvasive ventilation, and awake prone ventilation. Their role in patient management has been recently outlined, and instead of an early intubation strategy, represents gradual escalation of support that may be sufficient to treat hypoxemia and avoid the need for intubation and mechanical ventilation (Table 2).

In addition, the patient's hospital course was notable for the decline in known markers of active inflammation that mirrored the resolution of her hypoxemia and pneumonia. This included elevated lactate dehydrogenase, D-dimer, ferritin, and C-reactive protein with all but the latter rising and decreasing over 2 weeks. These findings provide additional information of the time for recovery and supports the use of these markers to monitor the course of pneumonia.

The patient declined all intervention, including oxygen, and recovered to her presumed prehospitalization condition. This experiment of nature due to unique circumstances may shed light on the natural time course of untreated hypoxemic COVID-19 pneumonia that has not previously been well appreciated. It is important to recognize that recovery occurred over 2 weeks. This is close to the observed and expected time for recovery that has been reported for those with severe COVID-19 pneumonia.

\section{CONCLUSIONS}

Since the emergence of the COVID-19, evidence has accumulated for the benefit of several adjunctive therapies in the treatment of this type of pneumonia, with corticosteroids providing a mortality benefit. Although unknown whether this patient's experience can be generalized to others or whether it represents her unique response, this case provides another perspective for comparison of treatments and reinforces the need for prospective, randomized clinical trials to establish treatment efficacy. The exact nature of silent hypoxemia of COVID-19 remains incompletely understood; however, this case highlights the importance of treating the individual instead of clinical markers and provides a time course for recovery from pneumonia and severe hypoxemia that occurs without oxygen or any other treatment over about 2 weeks.

\section{Author affiliations \\ Nicholas Hornstein is a Resident in the Department of Medi- cine, Division of General Internal Medicine, and Gilad Jaffe is a Fellow in the Department of Medicine, Division of Pulmonary, Critical Care and Sleep, both at Ronald Reagan-UCLA Medi- cal Center. Kelley Chuang is a Hospitalist in the Department of Medicine; Jaime Betancourt is the Director, Pulmonary Physiology Laboratory and Oxygen Program in the Pulmonary, Critical Care, and Sleep Section; and Guy Soo Hoo is the Chief, Pulmonary, Critical Care and Sleep Section; all at the Veterans Affairs Greater Los Angeles Healthcare System. Kelley Chuang is an Assistant Clinical Professor, Jaime Betancourt is an Associate Clinical Professor, and Guy Soo Hoo is a Clinical Professor, all at the David Geffen School of Medicine, Univer- sity of California Los Angeles.}

\section{Author disclosures}

The authors report no actual or potential conflicts of interest

\section{Disclaimer}

The opinions expressed herein are those of the authors and do not necessarily reflect those of Federal Practitioner, Frontline Medical Communications Inc., the US Government, or any of its agencies. This article may discuss unlabeled or investigational use of certain drugs. Please review the complete prescribing information for specific drugs or drug combinations - including indications, contraindications, warnings, and adverse effects-before administering pharmacologic therapy to patients.

\section{References}

1. Ioannou GN, Locke E, Green P, et al. Risk factors for hospitalization, mechanical ventilation, or death 
among 10131 US veterans with SARS-CoV-2 infection. JAMA Netw Open. 2020;3(9):e2022310. doi:10.1001/jamanetworkopen.2020.22310

2. Wiersinga WJ, Rhodes A, Cheng AC, Peacock SJ, Prescott HC. Pathophysiology, transmission, diagnosis, and treatment of coronavirus disease 2019 (COVID-19): a review. JAMA. 2020;324(8):782-793. doi:10.1001/jama.2020.12839

3. Alhazzani W, Moller MH, Arabi YM, et al. Surviving sepsis campaign: guidelines on the management of critically ill adults with coronavirus disease 2019 (COVID-19). Crit Care Med. 2020;48(6):e440-e469. doi:10.1097/CCM.0000000000004363

4. Ziehr DR, Alladina J, Petri CR, et al. Respiratory pathophysiology of mechanically ventilated patients with COVID-19: a cohort study. Am J Respir Crit Care Med. 2020;201(12):1560-1564. doi:10.1164/rccm.202004-1163LE

5. Wu Z, McGoogan JM. Characteristics of and important lessons from the coronavirus disease 2019 (COVID-19) outbreak in China: summary of a report of 72314 cases from the Chinese Center for Disease Control and Prevention. JAMA. 2020;323(13):1239-1242. doi:10.1001/jama.2020.2648

6. Zhou F, Yu T, Du R, et al. Clinical course and risk factors for mortality of adult inpatients with COVID-19 in Wuhan, China: a retrospective cohort study. Lancet. 2020;395 (10229):1054-1062. doi:10.1016/S01406736(20)30566-3

7. Tobin MJ, Laghi F, Jubran A. Why COVID-19 silent hypoxemia is baffling to physicians. Am J Respir Crit Care Med. 2020;202(3):356-360. doi:10.1164/rccm.202006-2157CP

8. Guan WJ, Ni ZY, Hu Y, et al. Clinical characteristics of coronavirus disease 2019 in China. N Engl J Med. 2020;382(18):1708-1720. doi:10.1056/NEJMoa2002032

9. Grasselli G, Zangrillo A, Zanella A, et al. Baseline characteristics and outcomes of 1591 patients infected with SARS-CoV-2 admitted to ICUs of the Lombardy Region, Italy. JAMA. 2020;323(16):1574-1581. doi:10.1001/jama.2020.5394

10. Raoof S, Nava S, Carpati C, Hill NS. High-flow, noninvasive ventilation and awake (nonintubation) proning in patients with coronavirus disease 2019 with respiratory failure. Chest. 2020;158(5):1992-2002. doi:10.1016/j.chest.2020.07.013

11. Ackermann M, Mentzer SJ, Jonigk D. Pulmonary vascular pathology in COVID-19. Reply. $N$ Engl $J$ Med. 2020;383(9):888-889. doi:10.1056/NEJMc2022068

12. McDonough G, Khaing P, Treacy T, McGrath C, Yoo EJ. The use of high-flow nasal oxygen in the ICU as a firstline therapy for acute hypoxemic respiratory failure secondary to coronavirus disease 2019. Crit Care Explor. 2020;2(10):e0257. doi:10.1097/CCE.0000000000000257

13. Hernandez-Romieu AC, Adelman MW, et al. Timing of intubation and mortality among critically ill coronavirus disease 2019 patients: a single-center cohort study. Crit Care Med. 2020;48(11):e1045-e1053. doi:10.1097/CCM.0000000000004600

14. Cummings MJ, Baldwin MR, Abrams D, et al. Epidemiology, clinical course, and outcomes of critically ill adults with COVID-19 in New York City: a prospective cohort study. Lancet. 2020;395(10239):1763-1770. doi:10.1016/S0140-6736(20)31189-2

15. Dhont S, Derom E, Van Braeckel E, Depuydt P, Lambrecht BN. The pathophysiology of 'happy' hypoxemia in COVID-19. Respir Res. 2020;21(1):198. doi:10.1186/s12931-020-01462-5

16. Wilkerson RG, Adler JD, Shah NG, Brown R. Silent hypoxia: a harbinger of clinical deterioration in patients with COVID-19. Am J Emerg Med. 2020;38(10):2243.e5-2243 .e6. doi:10.1016/j.ajem.2020.05.044

17. Gong J, Ou J, Qiu X, et al. A tool for early prediction of severe coronavirus disease 2019 (COVID-19): a multicenter study using the risk nomogram in Wuhan and Guangdong, China. Clin Infect Dis. 2020;71(15):833-840. doi:10.1093/cid/ciaa443

18. Force ADT, Ranieri VM, Rubenfeld GD, et al. Acute respiratory distress syndrome: the Berlin Definition. JAMA. 2012;307(23):2526-2533. doi:10.1001/jama.2012.5669

19. Marini JJ, Gattinoni L. Management of COVID-19 respiratory distress. JAMA. 2020;323(22):2329-2330. doi:10.1001/jama.2020.6825

20. Schaller T, Hirschbuhl K, Burkhardt K, et al. Postmortem examination of patients with COVID-19. JAMA. 2020;323(24):2518-2520. doi:10.1001/jama.2020.8907

21. Ackermann M, Verleden SE, Kuehnel M, et al. Pulmonary vascular endothelialitis, thrombosis, and angiogenesis in Covid-19. N Engl J Med. 2020;383(2):120-128. doi:10.1056/NEJMoa2015432

22. Wu C, Chen X, Cai $Y$, et al. Risk factors associated with acute respiratory distress syndrome and death in patients with coronavirus disease 2019 pneumonia in Wuhan, China. JAMA Intern Med. 2020;180(7):934-943. doi:10.1001/jamainternmed.2020.0994. Published correction appeared May 11, 2020. Errors in data and units of measure. doi:10.1001/jamainternmed.2020.1429

23. Yang J, Zheng Y, Gou X, et al. Prevalence of comorbidities and its effects in patients infected with SARS-CoV-2: a systematic review and meta-analysis. Int $J$ Infect Dis. 2020;94:91-95. doi:10.1016/j.jid.2020.03.017

24. Richardson S, Hirsch JS, Narasimhan M, et al. Presenting characteristics, comorbidities, and outcomes among 5700 patients hospitalized with COVID-19 in the New York City area. JAMA. 2020;323(20):2052-2059. doi:10.1001/jama.2020.6775

25. Tobin MJ, Jubran A, Laghi F. Misconceptions of pathophysiology of happy hypoxemia and implications for management of COVID-19. Respir Res. 2020;21(1):249. doi:10.1186/s12931-020-01520-y

26. Bickler PE, Feiner JR, Lipnick MS, McKleroy W. "Silent" presentation of hypoxemia and cardiorespiratory compensation in COVID-19. Anesthesiology. 2020;134(2):262-269. doi:10.1097/ALN.0000000000003578

27. Jounieaux V, Parreira VF, Aubert G, Dury M, Delguste $P$, Rodenstein DO. Effects of hypocapnic hyperventilation on the response to hypoxia in normal subjects receiving intermittent positive -pressure ventilation. Chest. 2002;121(4):1141-1148. doi:10.1378/chest.121.4.1141 